\title{
Existence of Positive Solutions of Nonlinear Second-Order Periodic Boundary Value Problems
}

\section{Ruyun Ma, Chenghua Gao, and Ruipeng Chen}

Department of Mathematics, Northwest Normal University, Lanzhou 730070, China

Correspondence should be addressed to Ruyun Ma,ruyun_ma@126.com

Received 31 August 2010; Revised 30 October 2010; Accepted 8 November 2010

Academic Editor: Irena Rachůnková

Copyright (C) 2010 Ruyun Ma et al. This is an open access article distributed under the Creative Commons Attribution License, which permits unrestricted use, distribution, and reproduction in any medium, provided the original work is properly cited.

This paper is devoted to study the existence of periodic solutions of the second-order equation $x^{\prime \prime}=$ $f(t, x)$, where $f$ is a Carathéodory function, by combining a new expression of Green's function together with Dancer's global bifurcation theorem. Our main results are sharp and improve the main results by Torres (2003).

\section{Introduction}

Let us say that the following linear problem:

$$
\begin{aligned}
& x^{\prime \prime}+a(t) x=0, \quad t \in(0, T), \\
& x(0)=x(T), \quad x^{\prime}(0)=x^{\prime}(T)
\end{aligned}
$$

is nonresonant when its unique solution is the trivial one. It is well known that (1.1), (1.2) is nonresonant then, provided that $h$ is a $L^{1}$-function, the Fredholm's alternative theorem implies that the inhomogeneous problem

$$
\begin{gathered}
x^{\prime \prime}+a(t) x=h(t), \quad t \in(0, T), \\
x(0)=x(T), \quad x^{\prime}(0)=x^{\prime}(T)
\end{gathered}
$$


always has a unique solution which, moreover, can be written as

$$
x(t)=\int_{0}^{T} G(t, s) h(s) d s,
$$

where $G(t, s)$ is the Green's function related to (1.1), (1.2).

In recent years, the conditions,

$\left(\mathrm{H}^{+}\right)$Problem (1.1), (1.2) is nonresonant and the corresponding Green's function $G(t, s)$ is positive on $[0, T] \times[0, T]$;

$\left(\mathrm{H}^{-}\right)$Problem $(1.1),(1.2)$ is nonresonant and the corresponding Green's function $G(t, s)$ is negative on $[0, T] \times[0, T]$,

have become the assumptions in the searching for positive solutions of singular second-order equations and systems; see for instance Chu and Torres [2], Chu et al. [3], Franco and Torres [4], Jiang et al. [5], and Torres [6]. Moreover, the positiveness of Green's function implies that an antimaximum principle holds, which is a fundamental tool in the development of the monotone iterative technique; see Cabada et al. [7] and Torres and Zhang [8].

The classical condition implying $\left(\mathrm{H}^{+}\right)$is an $L^{p}$-criteria proved in Torres [9] (and based on an antimaximum principle given in [8]). For the sake of completeness, let us recall the following result.

For any $1 \leq \alpha \leq \infty$, let $K(\alpha)$ be the best Sobolev constant in the inequality

$$
C\|u\|_{\alpha}^{2} \leq\|\dot{u}\|_{2}^{2}, \quad u \in \mathscr{H}:=H_{0}^{1}(0, T)
$$

given explicitly by (see [10])

$$
\begin{gathered}
K(\alpha)=\inf _{u \in \mathscr{L} \backslash\{0\}} \frac{\|\dot{u}\|_{2}^{2}}{\|u\|_{\alpha}^{2}}, \\
K(\alpha)= \begin{cases}\frac{2 \pi}{\alpha T^{1+2 / \alpha}}\left(\frac{2}{2+\alpha}\right)^{1-2 / \alpha}\left(\frac{\Gamma(1 / \alpha)}{\Gamma(1 / 2+1 / \alpha)}\right)^{2}, & 1 \leq \alpha<\infty, \\
\frac{4}{T}, & \alpha=\infty .\end{cases}
\end{gathered}
$$

Throughout the paper, "a.e." means "almost everywhere". Given $a \in L^{1}(0, T)$, we write $a>0$ if $a \geq 0$ for a.e. $t \in[0, T]$ and it is positive in a set of positive measure. Similarly, $a<0$ if $-a>0$.

Theorem A (see [9, Corollary 2.3]). Assume that $a \in L^{p}(0, T)$ for some $1 \leq p \leq \infty$ with $a>0$ and moreover

$$
\|a\|_{p}<K\left(2 p^{*}, T\right)
$$

with $1 / p+1 / p^{*}=1$. Then Condition $\left(H^{+}\right)$holds.

For the case that $a \prec 0$, Torres [9] proved the following. 
Theorem B (see [9, Theorem 2.2]). Assume that $a \in L^{p}(0, T)$ for some $1 \leq p \leq \infty$ with $a<0$. Then Condition $\left(\mathrm{H}^{-}\right)$holds. problem

To study the existence and multiplicity of positive solutions of the related nonlinear

$$
\begin{gathered}
x^{\prime \prime}+a(t) x=g(t, x), \quad t \in(0, T), \\
x(0)=x(T), \quad x^{\prime}(0)=x^{\prime}(T),
\end{gathered}
$$

it is necessary to find the explicit expression of $G(t, s)$.

Let $\varphi$ be the unique solution of the initial value problem

$$
\varphi^{\prime \prime}+a(t) \varphi=0, \quad \varphi(0)=1, \quad \varphi^{\prime}(0)=0,
$$

and let $\psi$ be the unique solution of the initial value problem

$$
\psi^{\prime \prime}+a(t) \psi=0, \quad \psi(0)=0, \quad \psi^{\prime}(0)=1
$$

Let

$$
D:=\varphi(T)+\psi^{\prime}(T)-2
$$

Atici and Guseinov [11] showed that the Green's function $G(t, s)$ of (1.1), (1.2) can be explicitly given as

$$
\begin{aligned}
G(t, s)= & \frac{\psi(T)}{D} \varphi(t) \varphi(s)-\frac{\varphi^{\prime}(T)}{D} \psi(t) \psi(s) \\
& + \begin{cases}\frac{\psi^{\prime}(T)-1}{D} \varphi(t) \psi(s)-\frac{\varphi(T)-1}{D} \varphi(s) \psi(t), & 0 \leq s \leq t \leq T, \\
\frac{\psi^{\prime}(T)-1}{D} \varphi(s) \psi(t)-\frac{\varphi(T)-1}{D} \varphi(t) \psi(s), & 0 \leq t \leq s \leq T .\end{cases}
\end{aligned}
$$

Torres [9] also studied the Green's function $G(t, s)$ of $(1.1),(1.2)$. Let $u$ be the unique solution of the initial value problem

$$
u^{\prime \prime}+a(t) u=0, \quad u(0)=0, \quad u^{\prime}(0)=1,
$$

and let $v$ be the unique solution of the initial value problem

$$
v^{\prime \prime}+a(t) v=0, \quad v(T)=0, \quad v^{\prime}(T)=-1
$$

Let

$$
\alpha:=\frac{1}{2+v^{\prime}(0)-u^{\prime}(T)} .
$$


Then the Green's function $K$ of (1.1), (1.2) given in [9] is in the form

$$
K(t, s)=\alpha[u(t)+v(t)]-\frac{1}{v(0)} \begin{cases}v(t) u(s), & 0 \leq s \leq t \leq T, \\ v(s) u(t), & 0 \leq t \leq s \leq T .\end{cases}
$$

However, there is a mistake in (1.16).

It is the purpose of this paper to point out that the Green's function in (1.16), which is induced by the two linearly independent solutions $u$ and $v$ of (1.13) and (1.14), should be corrected to the form

$$
G(t, s)=\frac{\alpha[u(s)+v(s)]}{v(0)}[u(t)+v(t)]-\frac{1}{v(0)} \begin{cases}v(t) u(s), & 0 \leq s \leq t \leq T, \\ v(s) u(t), & 0 \leq t \leq s \leq T .\end{cases}
$$

This will be done in Section 2. Finally in Section 3, we study the existence of one-sign solutions of the nonlinear problem

$$
\begin{gathered}
x^{\prime \prime}=f(t, x), \quad t \in(0, T), \\
x(0)=x(T), \quad x^{\prime}(0)=x^{\prime}(T) .
\end{gathered}
$$

The proofs of the main results are based on the properties of $G$ and the Dancer's global bifurcation theorem; see [12].

\section{Preliminaries}

Denote

$$
\begin{gathered}
\Lambda^{-}:=\left\{a \in L^{p}(0, T): a<0\right\}, \\
\Lambda^{+}:=\left\{a \in L^{p}(0, T): a>0,\|a\|_{p}<K\left(2 p^{*}\right) \text { for some } 1 \leq p \leq \infty\right\} .
\end{gathered}
$$

Recall that $u$ is a unique solution of IVP (1.13) and $v$ is a unique solution of IVP (1.14).

Lemma 2.1. Let $a \in L^{p}(0, T)$. Then

$$
u(T)=v(0)
$$

Proof. Since the Wronskian $W(u, v)(t)$ is constant, it follows that

$$
-u(T)=\left|\begin{array}{cc}
u(T) & v(T) \\
u^{\prime}(T) & v^{\prime}(T)
\end{array}\right|=\left|\begin{array}{cc}
u(t) & v(t) \\
u^{\prime}(t) & v^{\prime}(t)
\end{array}\right|=\left|\begin{array}{cc}
u(0) & v(0) \\
u^{\prime}(0) & v^{\prime}(0)
\end{array}\right|=-v(0) .
$$

The following result follows from the classical theory of Green's function. 
Lemma 2.2. Let $G(t, s)$ be the Green's function of (1.1), (1.2). Then

(i) $G:[0, T] \times[0, T] \rightarrow \mathbb{R}$ is continuous;

(ii) for a given $s \in(0, T), G(0, s)=G(T, s)$;

(iii) for a given $s \in(0, T), G_{t}(0, s)=G_{t}(T, s)$;

(iv) for a given $s \in(0, T), G(t, s)$ as a function of $t$ is a solution of (1.1) in the intervals $[0, s)$ and $(s, T]$.

Lemma 2.3. Let $a \in \Lambda^{+} \cup \Lambda^{-}$. Then the Green's function $G(t, s)$ induced by $u$ and $v$ is explicitly given by (1.17), that is,

$$
G(t, s)=\frac{u(s)+v(s)}{v(0)\left[2+v^{\prime}(0)-u^{\prime}(T)\right]}[u(t)+v(t)]-\frac{1}{v(0)} \begin{cases}v(t) u(s), & 0 \leq s \leq t \leq T, \\ v(s) u(t), & 0 \leq t \leq s \leq T .\end{cases}
$$

Remark 2.4. Notice that it is not necessary to assume that

$$
v(0) \neq 0 \text {. }
$$

In fact, if $a \in \Lambda^{+}$, then from [13, Remark in Page 3328], we have

$$
\underline{\lambda}_{1}(a) \geq\left(\frac{\pi}{T}\right)^{2}\left(1-\frac{\|a\|_{p}}{K\left(2 p^{*}\right)}\right)>0
$$

where $\underline{\lambda}_{1}(a)$ is the first eigenvalue of the antiperiodic boundary value problem

$$
x^{\prime \prime}+(\lambda+a(t)) x=0, \quad x(0)=-x(T), \quad x^{\prime}(0)=-x^{\prime}(T) .
$$

Now, by the same method to prove [8, Lemma 2.1], we may get that the solution $v$ of the IVP (1.14) has at most one zero in $[0, T]$. Since $v(T)=0$, we must have that $v(0) \neq 0$.

If $a \in \Lambda^{-}$, we claim that $v(t)>0$ for $t \in[0, T)$. Suppose on the contrary that there exists $\tau \in[0, T)$ such that

$$
v(\tau)=0, \quad v(t)>0, \quad \text { for } t \in(\tau, T)
$$

Then

$$
v^{\prime \prime}(t)=-a(t) v(t) \geq 0, \quad t \in[\tau, T)
$$

which means that

$$
v(t) \geq T-t, \quad t \in[\tau, T) .
$$

In particular, $v(\tau) \geq T-\tau>0, t \in[\tau, T)$. This is a contradiction. Therefore, $\delta=T$, and accordingly, $v(0) \geq 0$. 
Proof of Lemma 2.3. In the proof of [9, Proposition 2.0.1], the Green function was assumed to have the form

$$
K(t, s)=\alpha u(t)+\beta v(t)-\frac{1}{v(0)} \begin{cases}v(t) u(s), & 0 \leq s \leq t \leq T \\ v(s) u(t), & 0 \leq t \leq s \leq T\end{cases}
$$

However, for above $K(t, s)$, it is impossible to find constants $\alpha$ and $\beta$, such that

$$
K_{t}(0, s)=K_{t}(T, s), \quad s \in(0, T)
$$

So, we have to assume that the Green's function is of the form

$$
G(t, s)=\alpha(s) u(t)+\beta(s) v(t)-\frac{1}{v(0)} \begin{cases}v(t) u(s), & 0 \leq s \leq t \leq T, \\ v(s) u(t), & 0 \leq t \leq s \leq T .\end{cases}
$$

By Lemma 2.2 (ii), we have that $G(0, s)=G(T, s)$ for $s \in(0, T)$. Thus

$$
\beta(s) v(0)=G(0, s)=G(T, s)=\alpha(s) u(T), \quad s \in(0, T),
$$

which together with (2.2) imply that

$$
\beta(s)=\alpha(s), \quad s \in(0, T)
$$

From (2.13) and (2.15), we have

$$
G_{t}(t, s)=\alpha(s)\left[u^{\prime}(t)+v^{\prime}(t)\right]-\frac{1}{v(0)} \begin{cases}v^{\prime}(t) u(s), & 0 \leq s<t \leq T \\ v(s) u^{\prime}(t), & 0 \leq t<s \leq T\end{cases}
$$

and, for $s \in(0, T)$,

$$
G_{t}(0, s)=\alpha(s)\left[1+v^{\prime}(0)\right]-\frac{v(s)}{v(0)}, \quad G_{t}(T, s)=\alpha(s)\left[u^{\prime}(T)-1\right]+\frac{u(s)}{v(0)}
$$

Applying this and Lemma 2.2 (iii), it follows that

$$
\alpha(s)=\frac{u(s)+v(s)}{\left[2+v^{\prime}(0)-u^{\prime}(T)\right] v(0)} .
$$

Denote

$$
M:=\max _{0 \leq t, s \leq T} G(t, s), \quad m:=\min _{0 \leq t, s \leq T} G(t, s) .
$$


Finally, we state a result concerning the global structure of the set of positive solutions of parameterized nonlinear operator equations, which is essentially a consequence of Dancer [12, Theorem 2].

Suppose that $\mathbb{E}$ is a real Banach space with norm $\|\cdot\|$. Let $K$ be a cone in $\mathbb{E}$. A nonlinear mapping $A:[0, \infty) \times K \rightarrow \mathbb{E}$ is said to be positive if $A([0, \infty) \times K) \subseteq K$. It is said to be $K$ completely continuous if $A$ is continuous and maps bounded subsets of $[0, \infty) \times K$ to precompact subset of $\mathbb{E}$. Finally, a positive linear operator $V$ on $\mathbb{E}$ is said to be a linear minorant for $A$ if $A(\lambda, u) \geq \lambda V(x)$ for $(\lambda, u) \in[0, \infty) \times K$. If $B$ is a continuous linear operator on $\mathbb{E}$, denote $r(B)$ the spectrum radius of $B$. Define

$$
\mathrm{c}_{K}(B)=\{\lambda \in[0, \infty): \exists x \in K \text { with }\|x\|=1, x=\lambda B x\} .
$$

Lemma 2.5 (see [14, Lemma 2.1]). Assume that

(i) $K$ has a nonempty interior and $\mathbb{E}=\overline{K-K}$;

(ii) $A:[0, \infty) \times K \rightarrow E$ is $K$-completely continuous and positive, $A(\lambda, 0)=0$ for $\lambda \in \mathbb{R}$, $A(0, u)=0$ for $u \in K$, and

$$
A(\lambda, u)=\lambda B u+F(\lambda, u)
$$

where $B: \mathbb{E} \rightarrow \mathbb{E}$ is a strongly positive linear compact operator on $\mathbb{E}$ with $r(B)>0$, and $F$ : $[0, \infty) \times K \rightarrow \mathbb{E}$ satisfies $\|F(\lambda, u)\|=\circ(\|u\|)$ as $\|u\| \rightarrow 0$ locally uniformly in $\lambda$.

Then there exists an unbounded connected subset $\mathcal{C}$ of

$$
\Phi_{K}(A)=\{(\lambda, u) \in[0, \infty) \times K: u=A(\lambda, u), u \neq 0\} \cup\left\{\left(r(B)^{-1}, 0\right)\right\}
$$

such that $\left(r(B)^{-1}, 0\right) \in \mathcal{C}$.

Moreover, if $A$ has a linear minorant $V$, and there exists a

$$
(\mu, y) \in(0, \infty) \times K
$$

such that $\|y\|=1$ and $\mu V y \geq y$, then $\mathcal{C}$ can be chosen in

$$
\Phi_{K}(A) \cap([0, \mu] \times K) \text {. }
$$

\section{Main Results}

In this section, we consider the existence of positive solutions of nonlinear periodic boundary value problem

$$
\begin{gathered}
x^{\prime \prime}=f(t, x), \quad t \in(0, T), \\
x(0)=x(T), \quad x^{\prime}(0)=x^{\prime}(T),
\end{gathered}
$$

where $f:[0,1] \times \mathbb{R} \rightarrow \mathbb{R}$ is satisfying Carathéodory conditions. 


\section{1. $a \in \Lambda^{+}$}

By Theorem A, $a \in \Lambda^{+}$implies $G(t, s)>0$ on $[0, T] \times[0, T]$, and subsequently $M>m>0$. Let us define

$$
p^{+}:=\left\{x \in C[0, T] \mid x(t) \geq 0 \text { on }[0, T], \min _{t} x(t) \geq \frac{m}{M}\|x\|_{\infty}\right\}
$$

Lemma 3.1 (see [9, Theorem 3.2]). Let us assume that there exist $a \in \Lambda^{+}$and $0<r<R$ such that

$$
f(t, x)+a(t) x \geq 0, \quad \forall x \in\left[\frac{m}{M} r, \frac{M}{m} R\right], \text { a.e. } t \in[0, T] .
$$

Then (3.1) has a positive solution provided one of the following conditions holds

(i)

$$
\begin{aligned}
& f(t, x)+a(t) x \geq \frac{M}{T m^{2}} x, \quad \forall x \in\left[\frac{m}{M} r, r\right], \text { a.e. } t \in[0, T], \\
& f(t, x)+a(t) x \leq \frac{1}{T M} x, \quad \forall x \in\left[R, \frac{M}{m} R\right], \text { a.e. } t \in[0, T]
\end{aligned}
$$

(ii)

$$
\begin{gathered}
f(t, x)+a(t) x \leq \frac{1}{T M} x, \quad \forall x \in\left[\frac{m}{M} r, r\right], \text { a.e. } t \in[0, T] \\
f(t, x)+a(t) x \geq \frac{M}{T m^{2}} x, \quad \forall x \in\left[R, \frac{M}{m} R\right], \text { a.e. } t \in[0, T] .
\end{gathered}
$$

Let

$$
\begin{gathered}
r^{*}(t):=\frac{f(t,(m / M) r)+a(t)(m / M) r}{(m / M) r}, \quad \Gamma^{*}(t):=\frac{f(t,(M / m) R)+a(t)(M / m) R}{(M / m) R}, \\
\tilde{f}(t, x)= \begin{cases}\Gamma^{*}(t) x, & x \geq \frac{M}{m} R, \\
f(t, x)+a(t) x, & \frac{m}{M} r \leq x \leq \frac{M}{m} R, \\
r^{*}(t) x, & 0 \leq x \leq \frac{m}{M} r .\end{cases}
\end{gathered}
$$

Let

$$
\begin{array}{ll}
\underline{r}(t):=\min \left\{\frac{f(t, s)+a(t) s}{s} \mid s \in\left[\frac{m r}{M}, r\right]\right\}, & \bar{\Gamma}(t):=\max \left\{\frac{f(t, s)+a(t) s}{s} \mid s \in\left[R, \frac{M R}{m}\right]\right\}, \\
\bar{\gamma}(t):=\max \left\{\frac{f(t, s)+a(t) s}{s} \mid s \in\left[\frac{m r}{M}, r\right]\right\}, & \underline{\Gamma}(t):=\min \left\{\frac{f(t, s)+a(t) s}{s} \mid s \in\left[R, \frac{M R}{m}\right]\right\} .
\end{array}
$$


Theorem 3.2. Assume that

(A1) There exist $a \in \Lambda^{+} \cap C[0, T]$ and $0<r<R$ such that

$$
f(t, x)+a(t) x>0, \quad \forall x \in\left[\frac{m}{M} r, \frac{M}{m} R\right], \text { a.e. } t \in[0, T] .
$$

Then (3.1) has a positive solution provided one of the following conditions holds

(i) $\mu_{0}(\gamma)<1<\mu_{0}(\bar{\Gamma})$;

(ii) $\mu_{0}(\underline{\Gamma})<1<\mu_{0}(\bar{\gamma})$.

Here $\mu_{0}(\beta)$ denotes the principal eigenvalue of

$$
\begin{gathered}
x^{\prime \prime}+a(t) x=\mu \beta(t) x, \quad t \in(0, T), \\
x(0)=x(T), \quad x^{\prime}(0)=x^{\prime}(T) .
\end{gathered}
$$

Remark 3.3. Let $a \in \Lambda^{+}$and $\beta>0$. Then $\mu_{0}(\beta)>0$. Moreover, $\mu_{0}(\beta)$ is simple and the corresponding eigenfunction $\psi_{0} \in$ int $p^{+}$.

In fact, (3.10) is equivalent to

$$
x(t)=\mu \int_{0}^{T} G(t, s) \beta(s) x(s) d s:=\mu \AA x(t) .
$$

Since $G>0$ on $[0, T] \times[0, T]$, it follows that $\mathcal{A}\left(\mathcal{D}^{+}\right) \subset$ int $D^{+}$. From Krein-Rutman theorem, see $[15$, Theorem 19.3], we may get the desired results.

Remark 3.4. Theorem 3.2 is a partial generalization of Lemma 3.1. It is enough to prove that the condition (i) on $f$ in Theorem 3.2 holds when the condition (i) in Lemma 3.1 holds.

First, we claim that

(i) $\mu_{0}\left(M / \mathrm{Tm}^{2}\right)<1$;

(ii) $\mu_{0}(1 / T M)>1$.

To this end, let us denote by $\lambda_{0}$ the principal eigenvalue of the linear problem

$$
u^{\prime \prime}+a(t) u=\lambda u, \quad u(0)=u(T), \quad u^{\prime}(0)=u^{\prime}(T),
$$

and $\varphi$ the corresponding eigenfunction with $\varphi \in$ int $D^{+}$. Then applying the facts that $G \geq m$ and $G \not \equiv m$,

$$
\begin{aligned}
\lambda_{0} & =\mu_{0}\left(\frac{M}{T m^{2}}\right) \cdot \frac{M}{T m^{2}}, \\
\|\varphi\|_{\infty} & \geq \varphi(t) \\
& =\lambda_{0} \int_{0}^{T} G(t, s) \varphi(s) d s \\
& >\lambda_{0} m \frac{m}{M} T\|\varphi\|_{\infty^{\prime}}
\end{aligned}
$$


which together with (3.12), imply that

$$
\mu_{0}\left(\frac{M}{T m^{2}}\right)<1
$$

By the same method, with obvious changes, we may show that $\mu_{0}(1 / T M)>1$.

Now, we prove $\mu_{0}(\underline{\gamma})<1<\mu_{0}(\bar{\Gamma})$.

Define the operators $S_{1}, S_{2}: C[0, T] \rightarrow C[0, T]$ by

$$
\begin{aligned}
& \left(S_{1} u\right)(t)=\frac{M}{T m^{2}} \int_{0}^{T} G(t, s) u(s) d s, \\
& \left(S_{2} u\right)(t)=\int_{0}^{T} G(t, s) \underline{r}(s) u(s) d s,
\end{aligned}
$$

respectively.

Since $\underline{r}(t) \geq M / \operatorname{Tm}^{2}$, by [15, Theorem 19.3], we get $r\left(S_{2}\right) \geq r\left(S_{1}\right)$, where $r\left(S_{i}\right), i=1,2$, is the spectrum radius of $S_{i}$. Thus, $\mu_{0}(\gamma)=1 / r\left(S_{2}\right) \leq 1 / r\left(S_{2}\right)=\mu_{0}\left(M / \operatorname{Tm}^{2}\right)<1$.

Similarly, $\mu_{0}(\underline{\Gamma}) \geq \mu_{0}(1 / T M)>\overline{1}$.

Remark 3.5. The conditions $\mu_{0}(\underline{\gamma})<1<\mu_{0}(\bar{\Gamma})$ and $\mu_{0}(\underline{\Gamma})<1<\mu_{0}(\bar{\gamma})$ are optimal.

Let $\epsilon, \epsilon_{1}, \epsilon_{2}$ be positive constants with $\epsilon_{1}<\epsilon_{2}$, and

$$
\frac{1}{8}+\epsilon_{1} \leq a(t) \leq \frac{1}{8}+\epsilon_{2}
$$

Let us consider the problem

$$
u^{\prime \prime}+a(t) u=(a(t)+\epsilon) u, \quad u(0)=u(T), \quad u^{\prime}(0)=u^{\prime}(T) .
$$

Obviously, for $f(t, s)+a(t) s=(a(t)+\epsilon) s$, we have that

$$
\begin{gathered}
\underline{\gamma}(t)=\bar{\Gamma}(t)=a(t)+\epsilon, \\
\mu_{0}(\underline{\gamma})=\mu_{0}(\bar{\Gamma})=\mu_{0}(a(t)+\epsilon) .
\end{gathered}
$$

For $j=1,2$, the principal eigenvalue $\mu_{0}\left(1 / 8+\epsilon_{j}+\epsilon\right)$ of

$$
\begin{aligned}
x^{\prime \prime}+\left(\frac{1}{8}+\epsilon_{j}\right) x & =\mu \cdot\left(\frac{1}{8}+\epsilon_{j}+\epsilon\right) \cdot x, \quad t \in(0, T), \\
x(0) & =x(T), \quad x^{\prime}(0)=x^{\prime}(T)
\end{aligned}
$$


is

$$
\mu_{0}\left(\frac{1}{8}+\epsilon_{j}+\epsilon\right)=\frac{1+8 \epsilon_{j}}{8\left(\epsilon_{j}+\epsilon\right)+1}
$$

Applying the fact that

$$
\mu_{0}\left(\frac{1}{8}+\epsilon_{1}+\epsilon\right) \leq \mu_{0}(\underline{r})=\mu_{0}(\bar{\Gamma}) \leq \mu_{0}\left(\frac{1}{8}+\epsilon_{2}+\epsilon\right)
$$

though $\mu_{0}(\bar{\Gamma})$ is a little bit smaller than 1, the existence of positive solutions of (3.17) will not be guaranteed in this case.

Proof of Theorem 3.2. We only prove (i). (ii) can be proved by a similar method. problem

To study the existence of positive solutions of (3.1), let us consider the parameterized

$$
\begin{gathered}
x^{\prime \prime}+a(t) x=\mu \tilde{f}(t, x), \quad t \in(0, T), \\
x(0)=x(T), \quad x^{\prime}(0)=x^{\prime}(T) .
\end{gathered}
$$

Notice that

$$
\tilde{f}(t, x)=\gamma^{*}(t) x+\xi(t, x), \quad \tilde{f}(t, s)=\Gamma^{*}(t) s+\zeta(t, s),
$$

with

$$
\lim _{x \rightarrow 0} \frac{\xi(t, x)}{x}=0, \quad \lim _{s \rightarrow+\infty} \frac{\zeta(t, s)}{s}=0, \quad \text { a.e. } t \in[0, T]
$$

Thus, (3.22) can be rewritten as

$$
\begin{gathered}
x^{\prime \prime}+a(t) x=\mu \gamma^{*}(t) x+\mu \xi(t, x), \quad t \in(0, T), \\
x(0)=x(T), \quad x^{\prime}(0)=x^{\prime}(T) .
\end{gathered}
$$

Denote

$$
E=\left\{x \in C^{1}[0, T] \mid x(0)=x(T), x^{\prime}(0)=x^{\prime}(T)\right\}
$$

equipped with the norm $\|\cdot\|=\max \left\{\|x\|_{\infty},\left\|x^{\prime}\right\|_{\infty}\right\}$. Let

$$
\Phi^{+}:=\left\{x \in C^{1}[0, T] \mid x(t)>0 \text { on }[0, T], x(0)=x(T), x^{\prime}(0)=x^{\prime}(T)\right\} .
$$


From Lemma 2.5, there exists a continuum $C^{+}$of solutions of $(3.25)$ joining $\left(\mu_{0}\left(\gamma^{*}\right), 0\right)$ to infinity in $\Phi^{+}$. Moreover, $C^{+} \backslash\left\{\left(\mu_{0}\left(\gamma^{*}\right), 0\right)\right\} \subset \Phi^{+}$.

Now, we divide the proof into two steps.

Step 1. We show that $C^{+}$joining $\left(\mu_{0}\left(\gamma^{*}\right), 0\right)$ to $\left(\mu_{0}\left(\Gamma^{*}\right), \infty\right)$ in $\Phi^{+}$. So, $C^{+} \cap(\{1\} \times E) \neq \emptyset$, and accordingly, (3.25) has at least one positive solution $u$.

Suppose that $\left(\eta_{k}, y_{k}\right) \in C^{+}$with

$$
\left|\eta_{k}\right|+\left\|y_{k}\right\| \longrightarrow \infty
$$

We firstly show that $\left\{\eta_{k}\right\}$ is bounded.

In fact, it follows from the definition of $\tilde{f}$ and Condition (3.9) that

$$
\frac{\tilde{f}(t, s)}{s} \geq e(t), \quad \text { a.e. } t \in[0, T], s \in(0, \infty)
$$

for some $e \in L^{1}(0, T)$ with $e(t)>0$ a.e. on $[0, T]$.

We claim that $y_{k}$ has to change its sign in $[0, T]$ if $\eta_{k} \rightarrow \infty$.

In fact,

$$
y_{k}^{\prime \prime}(t)+a(t) y_{k}=\eta_{k} \frac{\tilde{f}\left(t, y_{k}\right)}{y_{k}} y_{k}
$$

yields that $y_{k}^{\prime \prime}(t)>0$ as $k$ is large enough. However, this contradicts the boundary condition $y_{k}^{\prime}(0)=y_{k}^{\prime}(T)$.

Therefore, $\left\{\eta_{k}\right\}$ is bounded.

Now, $\left\{\left(\eta_{k}, y_{k}\right)\right\}(k \in \mathbb{N})$ satisfy

$$
\begin{gathered}
y_{k}^{\prime \prime}+a(t) y_{k}=\eta_{k} \Gamma^{*}(t) y_{k}+\eta_{k} \zeta\left(t, y_{k}\right), \quad t \in(0, T) \\
y_{k}(0)=y_{k}(T), \quad y_{k}^{\prime}(0)=y_{k}^{\prime}(T)
\end{gathered}
$$

Let

$$
v_{k}:=\frac{y_{k}}{\left\|y_{k}\right\|}
$$

Then

$$
\begin{gathered}
v_{k}^{\prime \prime}+a(t) v_{k}=\eta_{k} \Gamma^{*}(t) v_{k}+\eta_{k} \frac{\zeta\left(t, y_{k}\right)}{y_{k}} v_{k}, \quad t \in(0, T), \\
v_{k}(0)=v_{k}(T), \quad v_{k}^{\prime}(0)=v_{k}^{\prime}(T) .
\end{gathered}
$$


Equation (3.33) is equivalent to

$$
v_{k}(t)=\eta_{k} \int_{0}^{T} G(t, s)\left(\Gamma^{*}(s) v_{k}(s)+\frac{\zeta\left(s, y_{k}(s)\right)}{y_{k}(s)} v_{k}(s)\right) d s
$$

Set

$$
w_{k}(t):=\Gamma^{*}(t) v_{k}(t)+\frac{\zeta\left(t, y_{k}(t)\right)}{y_{k}(t)} v_{k}(t)
$$

Since $\tilde{f}\left(t, y_{k}(t)\right)=\Gamma^{*}(t) y_{k}(t)+\zeta\left(t, y_{k}(t)\right)$, it follows from (3.7) and the fact $y_{k}>0$ on $[0, T]$ that

$$
\begin{aligned}
\left|\frac{\zeta\left(t, y_{k}(t)\right)}{y_{k}(t)}\right| & \leq\left|\Gamma^{*}(t)\right|+\left|\frac{\tilde{f}\left(t, y_{k}(t)\right)}{y_{k}(t)}\right| \\
& \leq\left|\Gamma^{*}(t)\right|+|\gamma(t)|+|\Gamma(t)|+|a(t)|+\max \left\{\left|\frac{f\left(t, y_{k}(t)\right)}{y_{k}(t)}\right|: \frac{m}{M} r \leq y_{k}(t) \leq \frac{M}{m} R\right\} \\
& \leq\left|\Gamma^{*}(t)\right|+|\gamma(t)|+|\Gamma(t)|+|a(t)|+\max \left\{\left|\frac{f(t, \tau)}{\tau}\right|: \frac{m}{M} r \leq \tau \leq \frac{M}{m} R\right\},
\end{aligned}
$$

which implies

$$
\left|\frac{\zeta\left(t, y_{k}(t)\right)}{y_{k}(t)}\right| \leq \sigma(t), \quad t \in(0, T)
$$

for some function $\sigma \in L^{1}[0, T]$, independent of $k$. Thus, it follows from (3.9) and (3.6) that $\left\{w_{k}(t)\right\}_{k=1}^{\infty}$ is bounded uniformly in $C[0, T]$. It is easy to check that $\left\{\eta_{k} \int_{0}^{T} G(t, s) w_{k}(s) d s\right\} \subset$ $C^{1}[0, T]$. This together with the fact that $C^{1}[0, T]$ imbeded compactly into $C[0, T]$ implies that, after taking a subsequence and relabeling if necessary, $v_{k} \rightarrow v^{*}$ in $C[0, T]$ for some $v^{*} \in C[0, T]$ and $\eta_{k} \rightarrow \eta^{*}$ for some $\eta^{*} \in[0, \infty)$, and using Lebesgue dominated convergence theorem, we get

$$
v^{*}(t)=\eta^{*} \int_{0}^{T} G(t, s) \Gamma^{*}(s) v^{*}(s) d s
$$

This implies that $v^{*} \in W^{2,1}(0, T)$ and

$$
\begin{gathered}
v^{* \prime \prime}+a(t) v^{*}=\eta^{*} \Gamma^{*}(t) v^{*}, \quad t \in(0, T), \\
v^{*}(0)=v^{*}(T), \quad v^{* \prime}(0)=v^{* \prime}(T),
\end{gathered}
$$


and subsequently,

$$
\eta^{*}=\mu_{0}\left(\Gamma^{*}\right)
$$

Therefore, $C^{+}$joins $\left(\mu_{0}\left(\gamma^{*}\right), 0\right)$ to $\left(\mu_{0}\left(\Gamma^{*}\right), \infty\right)$ in $\Phi^{+}$.

Step 2. We show that $u$ is actually a solution of (3.1).

To this end, we only prove that

$$
\begin{gathered}
x^{\prime \prime}+a(t) x=\tilde{f}(t, x), \quad t \in(0, T), \\
x(0)=x(T), \quad x^{\prime}(0)=x^{\prime}(T)
\end{gathered}
$$

has no positive solution $y$ with $\|y\|_{\infty}<r$ or $\|y\|_{\infty}>M R / m$.

In fact, suppose on the contrary that $y$ is a positive solution of (3.41) with $\|y\|_{\infty}<r$. Then we have from (3.7), (3.8) and the definition of $\tilde{f}$ that

$$
\frac{\tilde{f}(t, y(t))}{y(t)} \geq \underline{r}(t), \quad t \in[0, T] .
$$

Since

$$
\begin{aligned}
& y^{\prime \prime}(t)+a(t) y(t)=1 \cdot \frac{\tilde{f}(t, y(t))}{y(t)} y(t), \quad y(0)=y(T), \quad y^{\prime}(0)=y^{\prime}(T), \\
& w^{\prime \prime}(t)+a(t) w(t)=\mu_{0}(\underline{\gamma}) \cdot \underline{\gamma}(t) w(t), \quad w(0)=w(T), \quad w^{\prime}(0)=w^{\prime}(T),
\end{aligned}
$$

where $w$ is the corresponding eigenfunction of $\mu_{0}(\gamma)$ with $w>0$. Multiplying both sides of equation in (3.43) by $w$ and multiplying both sides of equation in (3.44) by $y$, integrating from 0 to $T$ and subtracting, we get

$$
\int_{0}^{T}\left[\frac{\tilde{f}(t, y(t))}{y(t)}-\mu_{0}(\underline{\gamma}) \cdot \underline{\gamma}(t)\right] y(t) w(t) d t=0,
$$

which together with (3.42) implies that

$$
\mu_{0}(\underline{r}) \geq 1
$$

However, this contradicts the assumption that $\mu_{0}(\gamma)<1$.

Next, suppose on the contrary that $y$ is a positive solution of (3.41) with $\|y\|_{\infty}>$ $M R / m$. Then we have from (3.7) and (3.8) and the definition of $\tilde{f}$ that

$$
\frac{\tilde{f}(t, y(t))}{y(t)} \leq \bar{\Gamma}(t), \quad t \in[0, T] .
$$


Since

$$
\begin{aligned}
y^{\prime \prime}(t)+a(t) y(t)=1 \cdot \frac{\tilde{f}(t, y(t))}{y(t)} y(t), \quad & y(0)=y(T), \quad y^{\prime}(0)=y^{\prime}(T), \\
z^{\prime \prime}(t)+a(t) z(t)=\mu_{0}(\bar{\Gamma}) \cdot \bar{\Gamma}(t) z(t), \quad z(0)=z(T), & z^{\prime}(0)=z^{\prime}(T),
\end{aligned}
$$

where $z$ is the corresponding eigenfunction of $\mu_{0}(\bar{\Gamma})$ with $z>0$. Multiplying both sides of the equation in (3.48) by $z$ and multiplying both sides of the equation in (3.49) by $y$, integrating from 0 to $T$ and subtracting, we get

$$
\int_{0}^{T}\left[\frac{\tilde{f}(t, y(t))}{y(t)}-\mu_{0}(\bar{\Gamma}) \cdot \bar{\Gamma}(t)\right] y(t) z(t) d t=0,
$$

which together with (3.47) implies that

$$
\mu_{0}(\bar{\Gamma}) \leq 1
$$

However, this contradicts the assumption that $\mu_{0}(\bar{\Gamma})>1$.

Let

$$
b(t):=\frac{f(t,-(m / M) r)-a(t)(m / M) r}{-(m / M) r}, \quad B(t):=\frac{f(t,-(M / m) R)-a(t)(M / m) R}{-(M / m) R},
$$

$$
\widehat{f}(t, x) \begin{cases}B(t) x, & x \leq-\frac{M}{m} R \\ f(t, x)+a(t) x, & -\frac{M}{m} R \leq x \leq-\frac{m}{M} r \\ b(t) x, & x \geq-\frac{m}{M} r .\end{cases}
$$

Let

$$
\begin{array}{ll}
\underline{b}(t):=\min \left\{\frac{f(t, s)+a(t) s}{s} \mid s \in\left[-r,-\frac{m r}{M}\right]\right\}, & \bar{B}(t):=\max \left\{\frac{f(t, s)+a(t) s}{s} \mid s \in\left[-\frac{M R}{m},-R\right]\right\}, \\
\bar{b}(t):=\max \left\{\frac{f(t, s)+a(t) s}{s} \mid s \in\left[-r,-\frac{m r}{M}\right]\right\}, & \underline{B}(t):=\min \left\{\frac{f(t, s)+a(t) s}{s} \mid s \in\left[-\frac{M R}{m},-R\right]\right\} .
\end{array}
$$

Similar to the proof of Theorem 3.2, we may prove the following. 
Theorem 3.6. Assume that

(H1) There exist $a \in \Lambda^{+} \cap C[0, T]$ and $0<r<R$ such that

$$
f(t, x)+a(t) x<0, \quad \forall x \in\left[-\frac{M}{m} R,-\frac{m}{M} r\right], \text { a.e. } t \in[0, T]
$$

Then (3.1) has a negative solution provided one of the following conditions holds

(i) $\mu_{0}(\underline{b})<1<\mu_{0}(\bar{B})$;

(ii) $\mu_{0}(\underline{B})<1<\mu_{0}(\bar{b})$.

\section{2. $a \in \Lambda^{-}$}

By Theorem B, $a \in \Lambda^{-}$implies $G(t, s)<0$ on $[0, T] \times[0, T]$, and subsequently $m<M<0$. Let us define

$$
p^{-}:=\left\{x \in C[0, T] \mid x(t) \geq 0 \text { on }[0, T], \min _{t} x(t) \geq \frac{M}{m}\|x\|_{\infty}\right\}
$$

Let $\mu_{0}(\beta)$ denote the principal eigenvalue of

$$
\begin{gathered}
x^{\prime \prime}+a(t) x=\mu \beta(t) x, \quad t \in(0, T), \\
x(0)=x(T), \quad x^{\prime}(0)=x^{\prime}(T) .
\end{gathered}
$$

Then, it is easy to see from Krein-Rutman theorem that $\mu_{0}(\beta)>0$ provided that $a \in \Lambda^{-}$and $\beta \prec 0$. Moreover, $\mu_{0}(\beta)$ is simple and the corresponding eigenfunction $\psi_{0} \in$ int $P^{-}$.

Let

$$
\begin{array}{ll}
\underline{q}(t):=\min \left\{\frac{f(t, s)+a(t) s}{s} \mid s \in\left[\frac{M r}{m}, r\right]\right\}, & \bar{Q}(t):=\max \left\{\frac{f(t, s)+a(t) s}{s} \mid s \in\left[R, \frac{m R}{M}\right]\right\}, \\
\bar{q}(t):=\max \left\{\frac{f(t, s)+a(t) s}{s} \mid s \in\left[\frac{M r}{m}, r\right]\right\}, & \underline{Q}(t):=\min \left\{\frac{f(t, s)+a(t) s}{s} \mid s \in\left[R, \frac{m R}{M}\right]\right\} .
\end{array}
$$

Applying the knowledge of the sign of Green's function when $a \in \Lambda^{-}$and the similar argument to prove Theorem 3.2 with obvious changes, we may prove the following.

Theorem 3.7. Let us assume that there exist $a \in \Lambda^{-} \cap C[0, T]$ and $0<r<R$ such that

$$
f(t, x)+a(t) x<0, \quad \forall x \in\left[\frac{M}{m} r, \frac{m}{M} R\right] \text {, a.e. } t \in[0, T]
$$


Then (3.1) has a negative solution provided one of the following conditions holds

(i) $\mu_{0}(\bar{q})<1<\mu_{0}(\underline{Q})$;

(ii) $\mu_{0}(\bar{Q})<1<\mu_{0}(\underline{q})$.

Let

$$
\begin{array}{ll}
\underline{p}(t):=\min \left\{\frac{f(t, s)+a(t) s}{s} \mid s \in\left[-r,-\frac{M r}{m}\right]\right\}, & \bar{P}(t):=\max \left\{\frac{f(t, s)+a(t) s}{s} \mid s \in\left[-\frac{m R}{M},-R\right]\right\}, \\
\bar{p}(t):=\max \left\{\frac{f(t, s)+a(t) s}{s} \mid s \in\left[-r,-\frac{M r}{m}\right]\right\}, & \underline{P}(t):=\min \left\{\frac{f(t, s)+a(t) s}{s} \mid s \in\left[-\frac{m R}{M},-R\right]\right\} .
\end{array}
$$

Theorem 3.8. Let us assume that there exist $a \in \Lambda^{-} \cap C[0, T]$ and $0<r<R$ such that

$$
f(t, x)+a(t) x>0, \quad \forall x \in\left[-\frac{m}{M} R,-\frac{M}{m} r\right] \text {, a.e. } t \in[0, T] .
$$

Then (3.1) has a negative solution provided one of the following conditions holds

(i) $\mu_{0}(\bar{p})<1<\mu_{0}(\underline{P})$;

(ii) $\mu_{0}(\bar{P})<1<\mu_{0}(\underline{p})$.

Remark 3.9. Very recently, Zhang [16] studied conditions on $a$ so that the operator $L_{a} x=$ $x^{\prime \prime}+a(t) x$ admits the maximum principle or the antimaximum principle with respect to the periodic boundary condition. By exploiting Green's functions, eigenvalues, rotation numbers, and their estimates, he gave several optimal conditions. The Green's function in Zhang [16] and the one in (2.4) are same. In fact, in the nonresonance case, Problem (1.1), (1.2) has a unique Green's function. In the resonance case, Problem (1.1), (1.2) has no Green's function any more.

Remark 3.10. It is worth remarking that Cabada and Cid [17], and Cabada et al. [18] have improved the $L^{p}$-criteria in Torres [9] to the case that $a$ may change its sign, and established the similar results for periodic one-dimensional $p$-Laplacian problems.

\section{Acknowledgments}

The authors are very grateful to the anonymous referees for their valuable suggestions. This paper is supported by the NSFC (no. 11061030), NWNU-KJCXGC-03-17, the Fundamental Research Funds for the Gansu Universities.

\section{References}

[1] W. Walter, Ordinary Differential Equations, vol. 182 of Graduate Texts in Mathematics, Springer, New York, NY, USA, 1998.

[2] J. Chu and P. J. Torres, "Applications of Schauder's fixed point theorem to singular differential equations," Bulletin of the London Mathematical Society, vol. 39, no. 4, pp. 653-660, 2007. 
[3] J. Chu, P. J. Torres, and M. Zhang, "Periodic solutions of second order non-autonomous singular dynamical systems," Journal of Differential Equations, vol. 239, no. 1, pp. 196-212, 2007.

[4] D. Franco and P. J. Torres, "Periodic solutions of singular systems without the strong force condition," Proceedings of the American Mathematical Society, vol. 136, no. 4, pp. 1229-1236, 2008.

[5] D. Jiang, J. Chu, and M. Zhang, "Multiplicity of positive periodic solutions to superlinear repulsive singular equations," Journal of Differential Equations, vol. 211, no. 2, pp. 282-302, 2005.

[6] P. J. Torres, "Weak singularities may help periodic solutions to exist," Journal of Differential Equations, vol. 232, no. 1, pp. 277-284, 2007.

[7] A. Cabada, P. Habets, and S. Lois, "Monotone method for the Neumann problem with lower and upper solutions in the reverse order," Applied Mathematics and Computation, vol. 117, no. 1, pp. 1-14, 2001.

[8] P. J. Torres and M. Zhang, "A monotone iterative scheme for a nonlinear second order equation based on a generalized anti-maximum principle," Mathematische Nachrichten, vol. 251, pp. 101-107, 2003.

[9] P. J. Torres, "Existence of one-signed periodic solutions of some second-order differential equations via a Krasnoselskii fixed point theorem," Journal of Differential Equations, vol. 190, no. 2, pp. 643-662, 2003.

[10] G. Talenti, "Best constant in Sobolev inequality," Annali di Matematica Pura ed Applicata. Serie Quarta, vol. 110, pp. 353-372, 1976.

[11] F. M. Atici and G. S. Guseinov, "On the existence of positive solutions for nonlinear differential equations with periodic boundary conditions," Journal of Computational and Applied Mathematics, vol. 132, no. 2, pp. 341-356, 2001.

[12] E. N. Dancer, "Global solution branches for positive mappings," Archive for Rational Mechanics and Analysis, vol. 52, pp. 181-192, 1973.

[13] M. Zhang and W. Li, "A Lyapunov-type stability criterion using $L^{\alpha}$ norms," Proceedings of the American Mathematical Society, vol. 130, no. 11, pp. 3325-3333, 2002.

[14] R. Ma, "Existence of positive solutions of a fourth-order boundary value problem," Applied Mathematics and Computation, vol. 168, no. 2, pp. 1219-1231, 2005.

[15] K. Deimling, Nonlinear Functional Analysis, Springer, Berlin, Germany, 1985.

[16] M. Zhang, "Optimal conditions for maximum and antimaximum principles of the periodic solution problem," Boundary Value Problems, Article ID 410986, 26 pages, 2010.

[17] A. Cabada and J. A. Cid, "On the sign of the Green's function associated to Hill's equation with an indefinite potential," Applied Mathematics and Computation, vol. 205, no. 1, pp. 303-308, 2008.

[18] A. Cabada, J. Á. Cid, and M. Tvrdý, "A generalized anti-maximum principle for the periodic one-dimensional $p$-Laplacian with sign-changing potential," Nonlinear Analysis: Theory, Methods $\mathcal{E}$ Applications, vol. 72, no. 7-8, pp. 3436-3446, 2010. 